\title{
Contactless power and data transmission for underwater sensor nodes
}

\author{
Yin-guo Huang ${ }^{*}$, Cheng Fang and Xing-fei Li
}

\begin{abstract}
When the technology of wireless sensor network is applied to ocean monitoring, the ocean underwater monitoring system will become a distributed network system. That could improve the level of ocean monitoring significantly. But most of the current ocean monitoring equipments work independently, and it is difficult to form them into a sensor network. To set up a sensor network, electric energy supply and data transmission are the chief problem, especially for the underwater sensor nodes. To solve this problem, a new method for contactless power and data transmission is proposed. It operates with the principle of electromagnetic coupling. Power and data are transmitted through a mutual channel. This channel is realized by the structure of a two-stage electromagnetic coupler. Here, steel rope is used as the closed steel ring to compose an electromagnetic coupler. Since steel rope is widely used in the current underwater equipments, the transmission channel of power and data can be realized without changing the existing structure. Furthermore, because of contactless transmission the sensor network has good expansibility and convenient configuration of its nodes for underwater applications. A voltage transformation model for the two-stage electromagnetic coupler is constructed and the transmitting principle of power and data is analyzed based on the model. According to the energy transmission efficiency and the bit error rate, an experimental prototype is designed and fabricated. Finally, the prototype testing of power transmission, data transmission, and reactive power compensation are carried out. The experimental results show that the proposed method for contactless power and data transmission is feasible and it is suitable for the application of underwater sensor nodes.
\end{abstract}

Keywords: Power and data transmission, Electromagnetic coupling, Sensor nodes, Reactive power compensation

\section{Introduction}

The ocean underwater monitoring system is a main part of ocean stereo monitoring network. It can obtain the data of ocean dynamics parameters and environmental elements, and it works in real-time, continuously and rapidly. That data include salinity, temperature, dissolved oxygen, chlorophyll, heavy metal, ocean current, and so on. The ocean underwater monitoring is characterized by the vast area and too many parameters. The monitoring system should meet the requirements of stereo monitoring with the characteristics of multipoint, multiple-profile, and network [1]. Therefore, it is important to introduce the technology of wireless sensor network (WSN) into the ocean monitoring system. With WSN, a distributed

\footnotetext{
* Correspondence: hyg@tju.edu.cn

State Key Laboratory of Precision Measuring Technology and Instruments, Tianjin University, Tianjin 300072, China
}

\section{Springer}

network system for ocean monitoring can be constructed. That will greatly improve the monitoring level [2].

The current monitoring systems usually operate in the mode of single-point or scanning, such as buoy, submersible buoy, underwater robot, shipborne equipments, and so on. When in scanning mode, the single-point equipment can realize a kind of multi-point and multiprofile monitoring by navigation. Although most of these equipments have the function of communication, they work as independent system and it is difficult to form them into a sensor network. As a sensor node, the underwater equipment has to realize the demand of long-term, networking and autonomous work. Therefore, power feeding and data transmission are the chief problem for this application. In this area, some related research works have been conducted. Because most of the current underwater equipments use batteries as the power, the 


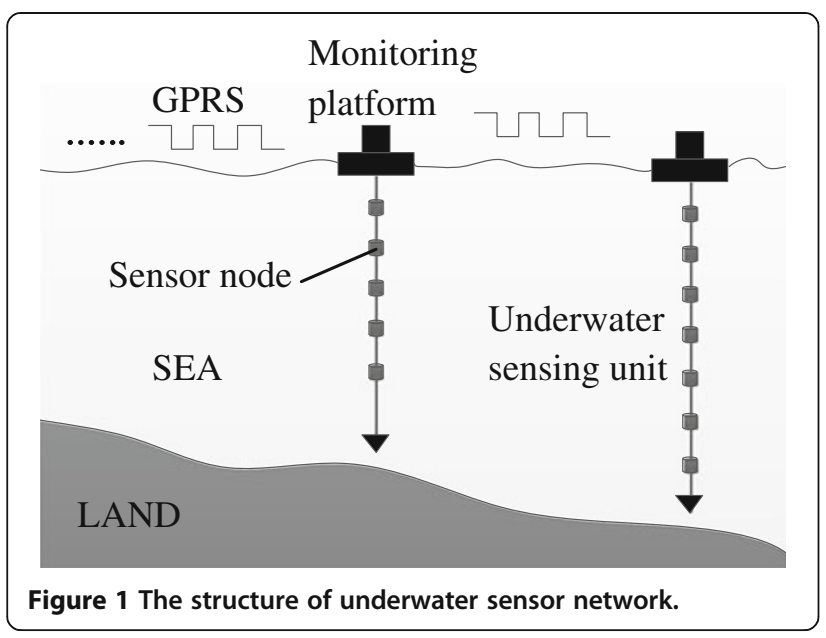

technology of low-power design gets much attention, such as the low-power underwater seismograph designed by Manuel et al. [3]. Tomisa et al. [4] utilized solar energy to power the ocean buoy with low maintenance cost. Ahnet al. [5] researched a new kind of buoy, in which wave energy was converted into electric power. Some other research on energy conversion for ocean

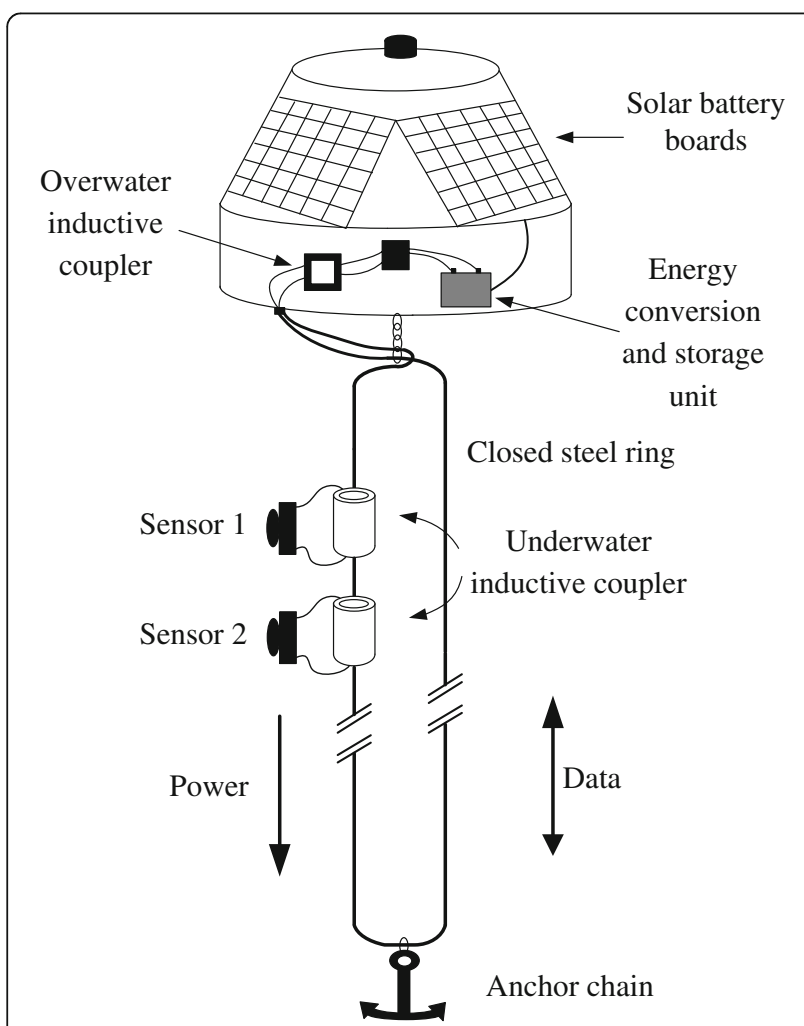

Figure 2 The cluster structure based on contactless power and data transmission.

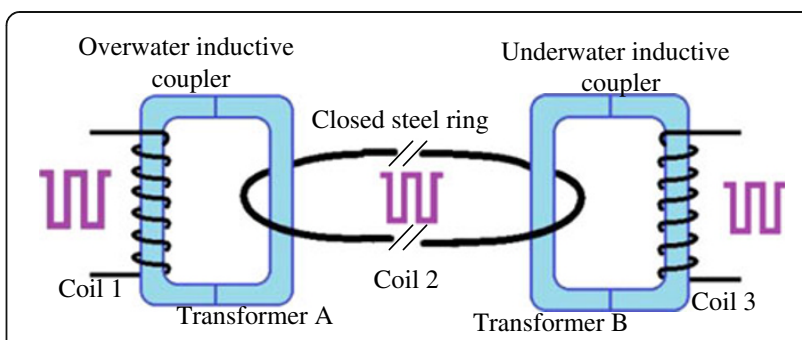

Figure 3 The schematic of contactless power and data transmission.

applications was also achieved, such as tidal energy [6] and ocean thermal energy conversion [7]. In underwater data transmission area, acoustic communication is the uppermost method at present [8]. Jane et al. [9] employed communication cables to transfer data between buoy and sensors.

This article proposes a new method for power feeding and data transmission based on electromagnetic coupling. It implements power and data transmission with a closed steel ring, which is widely used in the existing underwater equipments. Here, the closed steel ring serves as a transmission channel, and also a supporting structure to fasten the underwater sensors. Based on the proposed method, an experimental prototype is designed, which realizes contactless power and data transmission in the same channel for the underwater sensors.

\section{System structure}

\subsection{The structure of underwater sensor network}

As shown in Figure 1, the presented method is suitable for the underwater sensor network with a hierarchical topology structure, whose elementary unit is a cluster. This network is constructed on the basis of the common using structure of buoy. The cluster comprises a monitoring platform and a sensing unit. The overwater monitoring platform which communicates with other nodes using GPRS plays the role of backbone node. The underwater sensing unit includes many sensors, the so-called general nodes, which are configured along the direction of the closed steel ring. They can get the profile information at different depth. Therefore, an underwater distributed network can be built up by organizing network between clusters scatted in a specific ocean area.

\subsection{The cluster based on contactless power and data transmission}

Figure 2 gives the cluster structure with the function of contactless power and data transmission. It is constructed based on a typical architecture of buoy. 


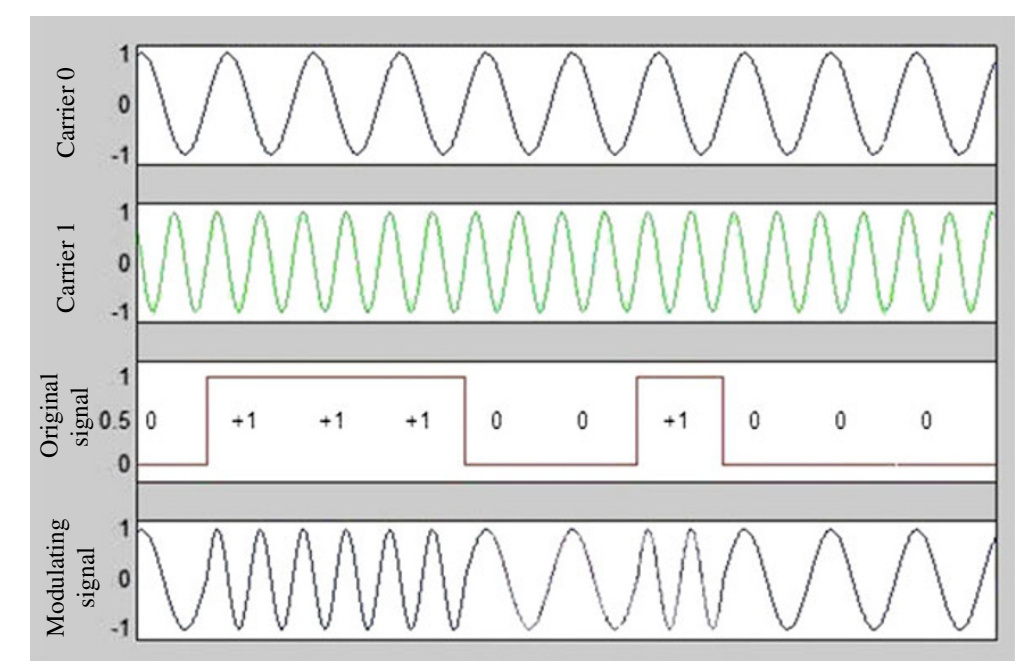

Figure 4 FSK modulation.

First, the overwater monitoring platform converts solar energy into electric power and stores it to power the whole cluster node. Power and data are transferred on the mutual channel between the monitoring platform and the underwater sensor. That channel is made up of overwater inductive coupler, closed steel ring, and underwater inductive coupler. Every sensor node fixed on the closed steel ring has an underwater inductive coupler, through which the sensor node obtains power supply and sends the measured data to the monitoring platform.

Because of electromagnetic coupling, there is no tight electrical contact between the closed steel ring and the sensor nodes. The sensor nodes can be fixed at arbitrary depth along the closed steel ring. It is easy to mount the sensor nodes. For instance, temperature sensor, salinity sensor, and ocean current sensor are arranged at different depth. That contributes to the good expansibility of the sensor network.

\section{The method for contactless power and data transmission}

\subsection{Transmission principle based on electromagnetic coupling}

The method for contactless power and data transmission is shown in Figure 3. Based on the principle of electromagnetic coupling, a structure of double-transformer is designed. The closed steel ring is not only the secondary coil of transformer A, but also the primary coil of transformer B.

According to the electromagnetic induction law, the input voltage $u_{i}$ of transformer $\mathrm{A}$ is given by

$$
u_{i}=N_{1} \frac{d \phi_{11}}{d t}=L_{1} \frac{d i_{1}}{d t}
$$

where $N_{1}$ is the number of turns of coil $1, L_{1}$ is the self-inductance of coil $1, \phi_{11}$ is the main flux of the coupler, and $i_{1}$ is the current flowing through coil 1.

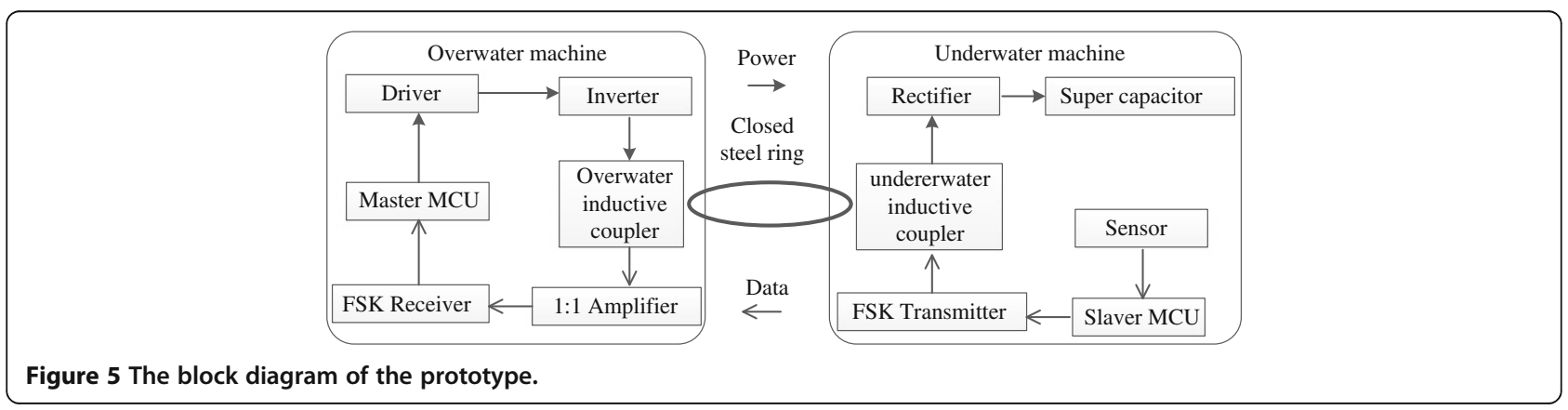




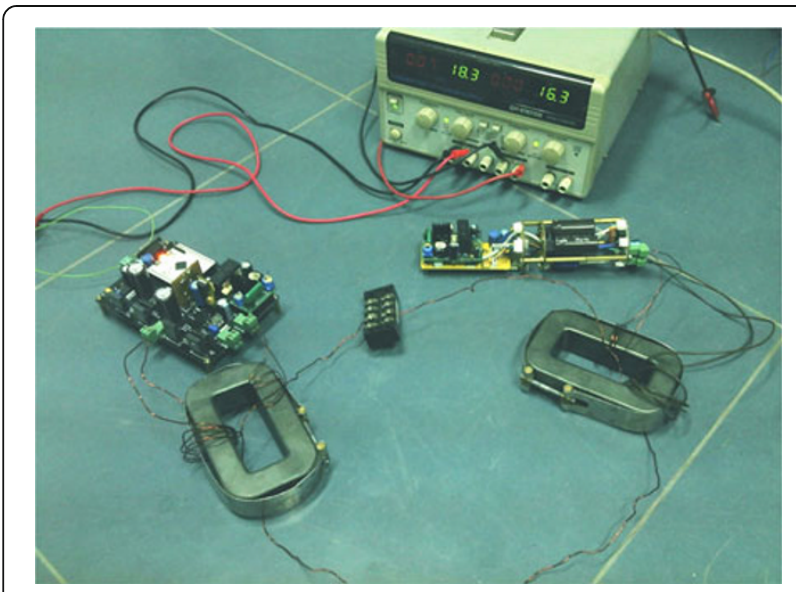

Figure 6 The picture of the prototype.

Two coils could not be fully coupled actually, and the coupling coefficient $k_{1}$ is

$$
k_{1}=\frac{M}{\sqrt{L_{1} L_{2}}}
$$

where $M$ is the mutual inductance of the two coils and $L_{2}$ is the self-inductance of coil 2. The relationship between the changes of magnetic flux in the two-layer coils is as follows:

$$
\frac{d \phi_{12}}{d t}=k_{1} \frac{d \phi_{11}}{d t}
$$

where $\phi_{12}$ is the flux of coil 2 . According to the above equation, the voltage $u_{2}$ of coil 2 can be obtained

$$
u_{2}=N_{2} \frac{d \phi_{12}}{d t}=N_{2} k_{1} \frac{d \phi_{11}}{d t}=k_{1} \frac{N_{2}}{N_{1}} u_{i}
$$

where $N_{2}$ is the number of turns of coil 2, and $N_{2}=1$ (single turn). Similarly, the voltage relationship of transformer B can be calculated. Finally, the output voltage $u_{3}$ is

$$
u_{3}=\frac{N_{3}}{N_{2}} u_{2}=k_{1} k_{2} \frac{N_{3}}{N_{1}} u_{i}
$$

Table 1 The results of power and data transmission experiments

\begin{tabular}{lll}
\hline Parameters & \multicolumn{2}{l}{ Transmission distance } \\
\cline { 2 - 3 } & $\mathbf{2 ~ \mathbf { ~ }}$ & $\mathbf{1 0 ~} \mathbf{~}$ \\
\hline Transmission efficiency & 0.78 & 0.33 \\
Bit error rate (\%) & 0.0 & 0.0 \\
\hline
\end{tabular}

where $N_{3}$ is the number of turns of coil 3 and $k_{2}$ is the coupling coefficient of transformer B.

\subsection{Implementation of power and data transmission}

Equation (5) gives the voltage transformation model of the two-stage coupler. That is the model for delivering electric energy. Both of the monitoring platform and underwater sensor should be powered with DC, while electromagnetic coupler needs AC. So, the energy delivery is a conversion process of DC/AC-AC/DC.

In Equation (5), $u_{\mathrm{i}}$ can be denoted as $u_{i}(t)=f(t) \cos$ $(\omega t)$, where $f(t)$ is the data to be sent. Accordingly, $u_{3}$ would include the information of $f(t)$. Here, the data signal is modulated by the carrier $\cos (\omega t)$ to fit the transmission channel. Consequently, the transmission distance can be tens of meters or even hundreds of meters by signal modulation.

In order to improve the anti-noise and anti-decay of underwater data transmission, the technology of frequency shift keying (FSK) is used. The signal to be delivered is modulated by carrier frequency. The working process is shown in Figure 4. The frequency of carrier 0 is lower than that of carrier 1 . After modulating, signals 0 and 1 in the original binary signal are, respectively, corresponded to carriers 0 and 1 . Finally, the modulating signal is received by the monitoring platform and then it is demodulated to revert to the original signal.

\section{Prototype design and experiments}

\subsection{Prototype design}

An experimental prototype is designed and Figure 5 gives its block diagram. It includes an overwater machine (monitoring platform) and an underwater machine (underwater sensor node). Both are controlled by MicroControllerUnit (MCU). The overwater machine has two kinds of work mode, power-sending and datareceiving. When in power-sending mode, the master-MCU generates sinusoidal pulse width modulation wave and sends it to the driver. Then the inverter generates sine wave under the control of the driver. Accordingly, the underwater machine works in power-receiving mode. That mode comprises two steps, rectification of the sine wave and charging the super capacitor. When the underwater machine works in data-sending mode, slave-MCU writes data to FSK transmitter. FSK transmitter outputs sine wave whose frequency is modulated. At the same time, the overwater machine receives the sine signal through a kind of 1:1 amplifier. Then the data demodulated by the FSK receiver are read out by the master-MCU.

In terms of Equation (5), the coupling coefficient has a great influence on the transmission efficiency. It is the key parameter to design the prototype. The 


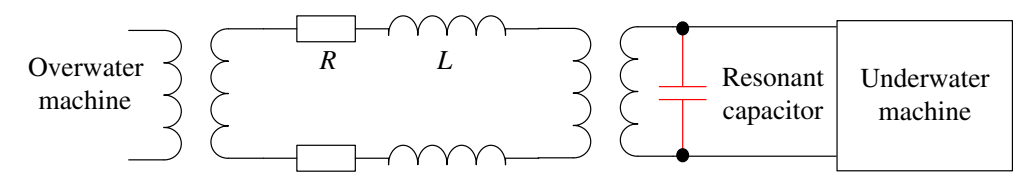

Figure 7 The transmission model of the prototype.

material and structure of the coupler determine the coupling coefficient.

The higher permeability of the coupler is conducive to the reduction of the magnetic leakage and the improvement of the coupling efficiency. The transmission efficiency is also related to the loss of the coupler. Different materials have different loss value per unit volume. Therefore, the electromagnetic coupler material should have such properties: (1) soft magnetic material, (2) small loss in highfrequency, (3) great initial permeability, and (4) great saturation magnetic induction. According to the above requirements, Febased nano-crystal material is selected to design the coupler.

For the convenience of fabricating, the shape of the coupler core is designed as rectangle. The area product method is used to design its size. With that method, there are several factors to be considered, among which the power is the most important factor. The area product method is given by

$$
A_{p}=\frac{P_{t} \times 10^{4}}{K_{f} K_{u} B_{m} J f}\left(\mathrm{~cm}^{4}\right)
$$

where $P_{\mathrm{t}}$ is the total apparent power, $B_{\mathrm{m}}$ is the magnetic flux density, $f$ is the operating frequency of the transformer, $K_{\mathrm{f}}$ is the waveform factor (4.0 for square wave, 4.44 for sine wave), $K_{\mathrm{u}}$ is the window utilization coefficient, and $J$ is the current density. For the same

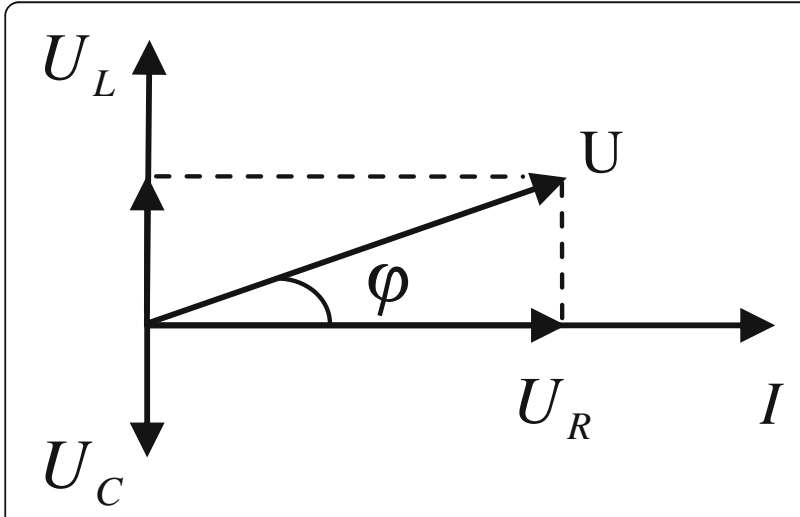

Figure 8 Reactive power compensation vector diagram. apparent power, increasing the window utilization factor $K_{\mathrm{u}}$, the working frequency $f$, the magnetic density $B_{\mathrm{m}}$, and current density $J$ can reduce the window area product, and then reduce the volume of the electromagnetic coupler. Similarly, for the same area product, increasing the above parameter values could improve the total apparent power of the electromagnetic coupler. The fabricated prototype is shown in Figure 6.

\subsection{Experiments on the prototype}

Changing transmission distance, power, and data transmission experiments are carried out. (1) The transmission distance is $2 \mathrm{~m}$. First, a high-power resistor is used as the load of the underwater machine. The overwater machine transmits electric energy to the underwater machine and the transmission efficiency is calculated. Then, under the same working conditions the underwater machine sends data while the overwater machine receives them. The bit error rate is calculated. (2) The transmission distance is $10 \mathrm{~m}$. Repeat the above experiments. Table 1 gives the experimental results.

Increasing the transmission distance, the bit error rate is the same while transmission efficiency decreases. Because other working conditions keep unchanged, it can be deduced that the decline of transmission efficiency is directly related to the transmission distance. Thereby, reactive power compensation experiment is carried out to improve the transmission efficiency. The transmission model of electric energy is shown in Figure 7. Between the overwater machine and the underwater machine, the impedance of the closed steel ring is replaced by resistors and inductors in series.

Due to the existence of the inductance, the prototype inevitably generates reactive power. In a pure inductance AC circuit shown in Figure 8, there is a lead phase $90^{\circ}$ between the phase of the voltage $U_{\mathrm{L}}$ and that of the current $I$. Accordingly, there are a phase lag of $90^{\circ}$ in the pure capacitance circuit and zero phase difference in the pure resistor circuit. According to the principle of the impedance transformation, a resonant capacitor is connected in parallel to the secondary coil of the underwater coupler. That is equivalent to the connection of a capacitor to the primary coil. By adjusting the capacitance value, the phase difference 


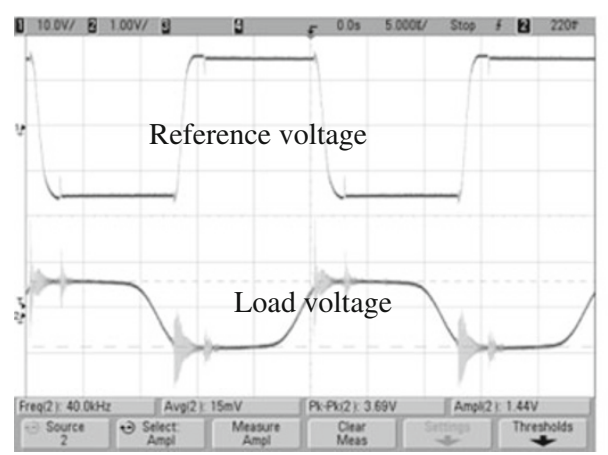

(a) Before compensation

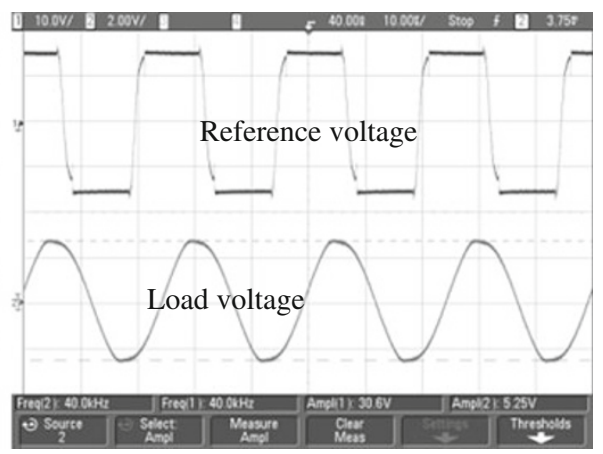

(b) After compensation

Figure 9 The load voltage comparison of before compensation and after compensation.

$\Delta \phi$ between the synthesized voltage $U$ and current $I$ can be reduced. When $\Delta \phi=0$, the resonance would occur. In the resonance state, the working frequency can be given by

$$
f=\frac{1}{2 \pi \sqrt{L C}}
$$

In the ideal condition, the power factor of the resonance circuit is 1 and the reactive power is zero.

When the transmission distance is $10 \mathrm{~m}$, the actual equivalent resistance and inductance of the closed steel ring are $0.5 \Omega$ and $200 \mu \mathrm{H}$, respectively. According to Equation (7), the theoretical value of the compensating capacitor is $1.15 \mu \mathrm{F}$ and here it takes a value of $1.0 \mu \mathrm{F}$. The transmission experiments on the compensated prototype are conducted once again with the transmission distance of $10 \mathrm{~m}$. Figure 9 shows the load voltage waveforms before and after compensation. The experimental results indicate that the transmission efficiency increases to 0.56 and the bit error rate still keeps a value of $0 \%$.

\subsection{Discussion}

The above experiments prove that the method for power and data transmission based on electromagnetic coupling is feasible. For data transmission, the bit error rate is $0 \%$ when the transmission distance is either 2 or $10 \mathrm{~m}$. That is due to good anti-interference ability of FSK modulation. If the transmission distance further increases, the bit error rate should still keep to a low level. For power transmission, the transmission efficiency declines with the increase of the transmission distance. Reactive power compensation plays an important role in improving the transmission efficiency. In addition, some other factors influence the transmission efficiency, such as the area of the closed steel ring, wattful loss, inverter efficiency, and the accuracy of the compensation model parameters. Those factors need further study. Therefore, it is feasible to further improve the transmission efficiency based on the present prototype.

\section{Conclusion}

The underwater sensor nodes should meet the requirements of long-term, networking and autonomous working. Therefore, the key to constructing a distributed sensor network is to solve the problem of power and data transmission. The principle of electromagnetic coupling is innovatively applied to contactless power and data transmission for underwater sensor nodes. With a two-stage electromagnetic coupler and closed steel ring structure, power and data are transmitted on the same channel. The voltage transformation model is established and the transmission process is analyzed. An experimental prototype is designed and fabricated. The results of test experiments prove that the presented method is feasible. This research provides a new method of power and data transmission for underwater sensor nodes. That method can directly be used in the existing underwater monitoring equipments, which will improve the ocean monitoring performance of real-time, maintenance-free, and expansibility.

\section{Competing interests}

The authors declare that they have no competing interests.

\section{Acknowledgement}

This study was supported by the National Natural Science Foundation of China (60972129) and the Science and Technology Support Project (State Key Laboratory of Mechatronical Engineering and Control).

Received: 6 January 2013 Accepted: 25 February 2013 Published: 20 March 2013 


\section{References}

1. HM Murphy, GP Jenkins, Observational methods used in marine spatial monitoring of fishes and associated habitats. A review. Mar. Freshw. Res 61(2), 236-252 (2010)

2. J Kong, JH Cui, DP Wu, M Gerla, Building underwater ad-hoc networks and sensor networks for large scale real-time aquatic applications, in Proceedings of IEEE Military Communications Conference (MILCOM 2005) (Atlantic City, USA, 2005), pp. 1535-1541

3. A Manuel, X Roset, J Del Rio, DM Toma, N Carreras, SS Panahi, A Garcia-Benadi, T Owen, J Cadena, Ocean bottom seismometer: design and test of a measurement system for marine seismology. Sensors 12(3), 3693-3719 (2012)

4. T Tomisa, S Krajcar, D Pinezic, Multipurpose marine buoy, in The 50th International Symposium ELMAR (ELMAR 2008) (Croatia, Zadar, 2008). pp. 401-405

5. KK Ahn, DQ Truong, HH Tien, J Yoon, An innovative design of wave energy converter. Renew. Energy 42(2), 186-194 (2012)

6. YS Cho, JW Lee, W Jeong, The construction of a tidal power plant at Sihwa Lake, Korea. Energy Sources 34(14), 1280-1287 (2012)

7. S Goto, Y Motoshima, T Sugi, Construction of simulation model for OTEC plant using Uehara cycle. Electric. Eng. Jpn. 176(2), 1-13 (2011)

8. W Zhu, M Zhu, Y Wu, B Yang, L Xu, X Fu, F Pan, Signal processing in underwater acoustic communication system for manned deep submersible "Jiaolong". J. Acoust. Soc. Am 131(4), 3238 (2012)

9. T Jane, R Christopher, G Antonio, K Taimur, F Steve, H lan, B Nathan, V Chris $\mathrm{M}$ lan, Real world issues in deploying a wireless sensor network for oceanography, inWorkshop on Real-World Wireless Sensor Networks (RWWSN, Stockholm. Sweden 2005, 307-322 (2005)

doi:10.1186/1687-1499-2013-81

Cite this article as: Huang et al:: Contactless power and data transmission for underwater sensor nodes. EURASIP Journal on Wireless Communications and Networking 2013 2013:81.

\section{Submit your manuscript to a SpringerOpen ${ }^{\circ}$ journal and benefit from:}

- Convenient online submission

- Rigorous peer review

- Immediate publication on acceptance

- Open access: articles freely available online

- High visibility within the field

- Retaining the copyright to your article 